\title{
Proteomics reveals the role of Eucommia ulmoides-Tribulus terrestris in the central regulation of antihypertension by improving the hypothalamus in spontaneously hypertensive rats
}

\author{
Peng Zhang ${ }^{1 \#}$, Jiacheng Zhang ${ }^{2 \#}$, Jianlian $W^{2 *}{ }^{2 \#}$, Shuai Feng ${ }^{3}$, Yingzi Qi ${ }^{4}$, Chuanhua Yang ${ }^{2}$, Yuehua Jiang ${ }^{5}$ \\ ${ }^{1}$ First Clinical Medical College, Shandong University of Traditional Chinese Medicine, Jinan, China; ${ }^{2}$ Department of Cardiovascular, Affiliated \\ Hospital of Shandong University of Traditional Chinese Medicine, Jinan, China; ${ }^{3}$ School of Pharmacy, Shandong University of Traditional Chinese \\ Medicine, Jinan, China; ${ }^{4}$ Health College, Shandong University of Traditional Chinese Medicine, Jinan, China; ${ }^{5}$ Department of Central Laboratory, \\ Affiliated Hospital of Shandong University of Traditional Chinese Medicine, Jinan, China \\ Contributions: (I) Conception and design: P Zhang, Y Jiang; (II) Administrative support: J Zhang; (III) Provision of study materials or patients: S \\ Feng; (IV) Collection and assembly of data: Y Qi; (V) Data analysis and interpretation: P Zhang, J Zhang; (VI) Manuscript writing: All authors; (VII) \\ Final approval of manuscript: All authors. \\ "These authors contributed equally to this work. \\ Correspondence to: Prof. Yuehua Jiang. Department of Central Laboratory, Affiliated Hospital of Shandong University of Traditional Chinese \\ Medicine, No. 16369, Jingshi Road, Jinan 250011, China. Email: jiang_yuehua@hotmail.com.
}

Background: In this study, the combination of Eucommia ulmoides-Tribulus terrestris (EU-TT) was used to intervene in aging spontaneously hypertensive rats (SHRs). Quantitative proteomics sequencing was performed to screen the targets of EU-TT, so as to provide data support for the clinical application of EUTT.

Methods: Eighteen-month-old SHRs were administered EU-TT $(5.53 \mathrm{~g} / \mathrm{kg} / \mathrm{day})$ intragastrically for 8 weeks. Blood pressure was recorded to evaluate the efficacy of EU-TT in vivo. Transmission electron microscopy (TEM) and hematoxylin-eosin (HE) staining were used to assess the morphology of the hypothalamus. The label free proteomics assay was performed to screen the targets of EU-TT in hypertensive hypothalami. ERK, JNK, and p38 were chosen for Reverse Transcription-quantitative Polymerase Chain Reaction (RT-qPCR) and Western blot.

Results: After 8 weeks of treatment, EU-TT effectively decreased systolic blood pressure (SBP) by $19.2 \mathrm{mmHg}$ and diastolic blood pressure (DBP) by $8.6 \mathrm{mmHg}(\mathrm{P}<0.05)$, and improved the hypothalamus morphology of aging SHRs. Label free proteomics identified 248 differentially expressed (DE) proteins (157 were upregulated and 91 were downregulated) in the hypothalamus after EU-TT treatment. Kyoto Encyclopedia of Genes and Genomes (KEGG) pathway analysis suggested that EU-TT regulated the MAPK signal transduction pathway, which was also confirmed by RT-qPCR and Western blot.

Conclusions: EU-TT steadily decreased the SBP and DBP of aging SHRs, and improved the morphology of the hypothalamus, which was pharmacologically related to the MAPK signaling pathway.

Keywords: Aging spontaneously hypertensive rats (SHRs); Eucommia ulmoides-Tribulus terrestris (EU-TT); hypothalamus; label free proteomics; MAPK signaling pathway

Submitted Nov 05, 2021. Accepted for publication Jan 07, 2022.

doi: 10.21037/atm-21-6390

View this article at: https://dx.doi.org/10.21037/atm-21-6390 


\section{Introduction}

Hypertension is one of the most common chronic diseases. In the elderly over 80 years old, the prevalence of hypertension is close to $90 \%$. Hypertension is the primary risk factor for stroke, myocardial infarction, and cardiovascular death (1). The foundational cause of isolated systolic hypertension (ISH) in the elderly lies in progressive arteriosclerotic vascular wall stiffness and the retrogression of the central regulation of blood pressure (2). As an important control area of aging, the hypothalamus is known as the "aging clock" of higher animals and humans. At the same time, also an earlier aging part in the process of brain aging, which is significantly earlier than the thalamus, hippocampus, somatic cortex, forebrain and plays an important regulatory role in the development of aging (3). One of the specific manifestations of hypothalamic aging is the degenerative regulatory capacity on blood pressure $(4,5)$.

Several studies have confirmed the effect of Eucommia ulmoides-Tribulus terrestris (EU-TT) on lowering blood pressure, improving diuresis, lowering blood lipid levels, anti-arteriosclerosis, reducing left ventricular remodeling, and improving early cardiac function after myocardial infarction (6). Our previous studies confirmed the endothelial protective effects of Tribulus terrestris both in vivo and in vitro $(7,8)$. The combined use of EU-TT demonstrated an overall regulatory effect on the whole body and also alleviated hypertensive target organ damage and exhibited an enhanced antihypertensive effect in elderly spontaneously hypertensive rats (SHRs) (9-11). However, the pharmacological mechanism of EU-TT needs to be further studied. From the perspective of proteomics, this study aims to explore the target of EU-TT in the hypothalamus of elderly SHRs, so as to better understand the pharmacodynamic effect of EU-TT on the central regulation of blood pressure for improving ISH in the elderly.

We present the following article in accordance with the ARRIVE reporting checklist (available at https://dx.doi. org/10.21037/atm-21-6390).

\section{Methods}

\section{Preparation of EU-TT}

The EU-TT was purchased from Affiliated Hospital of Shandong University of Traditional Chinese Medicine (Jinan, China), which was boiled with deionized water $(1: 10$, then $1: 5, \mathrm{w} / \mathrm{v})$ twice for 30 minutes. The solution was concentrated to $1.5 \mathrm{~g}$ raw herbs/mL, through a rotary vacuum evaporator $\left(70^{\circ} \mathrm{C}\right)$, after filtration.

\section{Animals}

Animal welfare and experimental steps were executed in accordance with the principles of "Laboratory animalGuidelines for ethical review of animal welfare (GB/ T 35892-2018)" (General Administration of Quality Supervision, Inspection and Quarantine of the People's Republic of China, 2018), and approved by the Faculty of Animal Ethics Committee of Affiliated Hospital of Shandong University of Traditional Chinese Medicine (Jinan, China) (No. 2020-08). A protocol was prepared before the study without registration. A total of 20 18-month-old SHRs [specific pathogen free (SPF) level, weighing 320-350 g] and 10 18-month-old Wistar-Kyoto (WKY) rats were purchased from Beijing Vital River Laboratory Animal Technology Co., Ltd. [certificate: SCXC (Jing) 2016-0006]. All rats were placed in an air-conditioned house with a $12 \mathrm{~h} / 12 \mathrm{~h} \mathrm{light/dark}$ cycle at a temperature of $21 \pm 1{ }^{\circ} \mathrm{C}$, humidity of $50 \% \pm 5 \%$ and had access to food and water ad libitum. All rats were fed with a standard chow diet (consisting of $11.5 \%$ energy as fat, $20.8 \%$ as protein, and $67.7 \%$ as carbohydrates, with $0.5 \% \mathrm{NaCl}$, Lukang, Jining, China) for 8 weeks. We recorded weight and blood pressure every 2 weeks, and used ALC-NIBP noninvasive blood pressure analysis system to measure systolic blood pressure (SBP) and diastolic blood pressure (DBP). We repeated the measurements 5 times in parallel, and then recorded the average values of SBP and DBP.

After 1 week of adaptation, 20 SHRs were randomly divided into 2 groups to confirm the efficacy of EU-TT, including the EU-TT group [our previous study (10) suggested that crude drug concentration was $5.53 \mathrm{~g} / \mathrm{kg} / \mathrm{d}$, for a duration of 8 weeks] and the SHR group. A total of $10 \mathrm{WKY}$ rats were used as the control group $(\mathrm{n}=10)$. The WKY group and SHR group were perfused with the same physiological saline.

\section{Tissue collection}

Rats were sacrificed after treatment for 8 weeks. After anesthesia with sodium pentobarbital ( $40 \mathrm{mg} / \mathrm{kg}$, i.p.), the hypothalami were removed on ice as quickly as possible. The hypothalami were analyzed by the label free proteomics assay, hematoxylin-eosin (HE) staining, transmission electron microscopy (TEM), transcriptome sequencing, 
reverse transcription-quantitative polymerase chain reaction (RT-qPCR), and Western blotting.

\section{TEM}

The hypothalamus tissue was immediately put into $2.5 \%$ glutaraldehyde solution. Brain micromorphology was observed under TEM $(\times 15,000)(J E O L-1200$, JEOL, Japan).

\section{HE staining}

HE staining (Solarbio, Beijing, China) stained the hypothalamic. The optical microscope (Carl Zeiss, Germany) was used to photograph the stained hypothalamic.

\section{Label free proteomics assay}

Protein was extracted from the tissue, then the protein concentration of the sample was measured by the Bradford method. After protein quantification, $200 \mu \mathrm{g}$ protein solution was placed in a centrifuge tube, then $25 \mathrm{mM}$ DTT was added to the final concentration and reacted at $60{ }^{\circ} \mathrm{C}$ for $1 \mathrm{~h}$. To this final concentration, $50 \mathrm{mM}$ iodoacetamide was added at room temperature for 10 minutes. The reduced alkylated protein solution was added into a $10 \mathrm{~K}$ ultrafiltration tube, centrifuged for 20 minutes at 12,000 rpm, and the solution at the bottom of the collection tube was discarded. The solution buffer $(100 \mu \mathrm{L})$ was then added. After centrifugation at $12,000 \mathrm{rpm}$ for 20 minutes, the solution at the bottom of the collection tube was discarded, which was repeated 3 times. The collection tube was replaced with a new one, and trypsin was added into the ultrafiltration tube, with a total amount of $4 \mu \mathrm{g}$ (mass ratio to protein 1:50) for a volume of $50 \mu \mathrm{L}$. The reaction was incubated at $37^{\circ} \mathrm{C}$ overnight. The next day, it was centrifuged at 12,000 rpm for 20 minutes, and the peptide solution after enzymatic digestion was centrifuged at the bottom of the collection tube.

Subsequently, $50 \mu \mathrm{L}$ solution buffer was added into the ultrafiltration tube for 12,000 revolutions, and after centrifugation again for 20 minutes, combined with the previous step, $100 \mu \mathrm{L}$ was obtained at the bottom of the collection tube. Protein analysis was performed by nano upgraded reversed phase chromatography-Q Exactive. The selected database was the Swissprot library. Mass spectrometry was performed by Thermo Q-Exactive mass spectrometry.

\section{RT-qPCR}

After extraction with TRIzol reagent (Invitrogen Corp, Carlsbad, CA, USA) and quantification with a NanoDrop 2000c spectrophotometer (Thermo Fisher Scientific, Waltham, MA, USA), total RNA samples were reversetranscribed using the Sparkjade Reverse Transcriptase Kit (Sparkjade, Qingdao, China). The relevant RNA expression levels were determined using the $2^{-\Delta \Delta \mathrm{Ct}}$ method. Fold change was normalized to that of the WKY group. $\beta$-actin was used as the internal reference.

The primer (Sangon Biotech, Jinan, China) sequences were: ERK1: 5 '-CCAAATCTGACTCCAAAGC3 ' / 5 ' - TCCATGTCAAGGTGAATG-3 '; p 38 : $5^{\prime}$ - A GTGGCTGA C C C T TAT TAC - 3 ' / 5 ' CACAGTGAAGTGGGATGGA-3'; and JNK: 5 ' - AGGGAGCACACAATAGAGGAGTG - 3 ' / 5 ' GAAGACGACGGATGCTGAGAG-3'.

\section{Western blotting analysis}

For validation of the data from the proteomics assay, key proteins of the MAPK signaling pathway such as p-ERK, p-p38, and p-JNK were chosen for the Western blot assay.

Protein was extracted from hypothalamus tissues in RIPA lysis buffer (SparkJade, EA0002, Jinan, China). In short, a total of $30 \mu \mathrm{g}$ protein samples were electrophoresed. After being transferred onto PVDF membranes, the blots were incubated with horseradish peroxidase conjugated to goat anti-rabbit IgG $(1: 20,000)$ on ice for $1 \mathrm{~h}$, and visualized by incubation with Immobilon Western Chemiluminescent HRP Substrate (Merck Millipore, 1579205, Darmstadt, Germany). The bands were quantified by Image J (National Institutes of Health, Bethesda, Maryland, USA) and the density value was normalized to that of $\beta$-actin.

\section{Statistical analysis}

SPSS 26.0 was used for statistical analysis. The collected data were expressed as mean \pm standard deviation (SD). One way ANOVA was used for statistical analysis, followed by LSD test. $\mathrm{P}<0.05$ was considered as statistically significant.

\section{Results}

\section{EU-TT decreases blood pressure in aging SHRs}

The data (Figure 1) confirmed that EU-TT administration decreased SBP by $19.2 \mathrm{mmHg}$ and DBP by $8.6 \mathrm{mmHg}$ in 

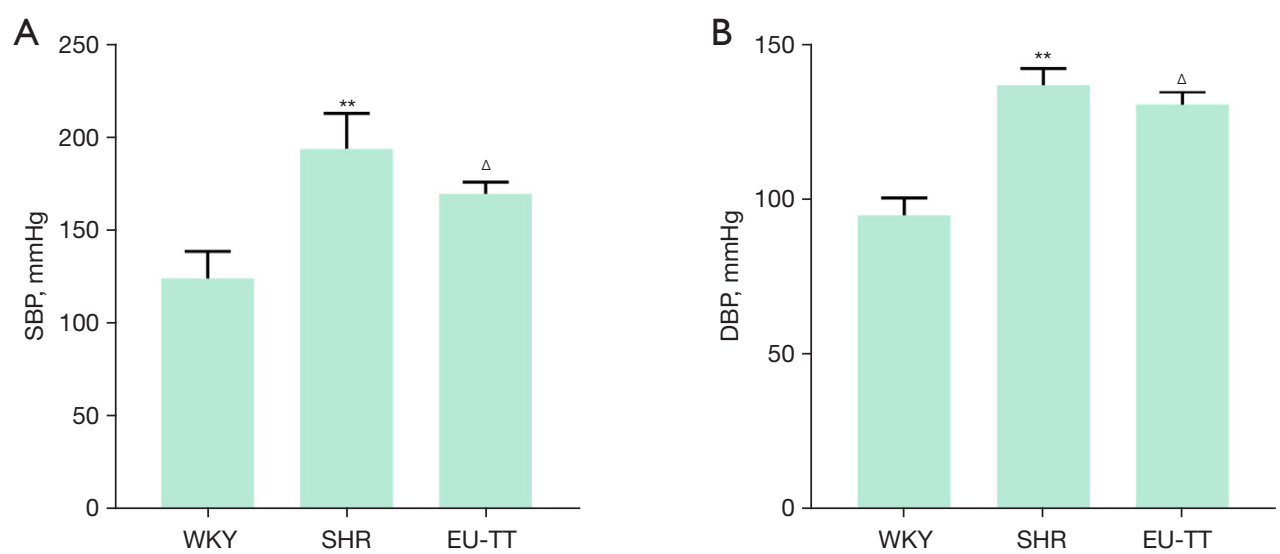

Figure 1 EU-TT attenuates blood pressure in aging SHRs. (A) Systolic pressure $(\mathrm{n}=10)$; (B) diastolic pressure $(\mathrm{n}=10)$. ${ }^{* *} \mathrm{P}<0.01$ vs. the WKY group; ${ }^{\wedge} \mathrm{P}<0.05$ vs. the SHR group. WKY, Wistar Kyoto rats; SHRs, spontaneously hypertensive rats; EU-TT, Eucommia ulmoides-Tribulus terrestris; SBP, systolic blood pressure; DBP, diastolic blood pressure.

aging SHRs $(P<0.01)$.

\section{EU-TT improves hypertension-related morphology of the bypothalamus}

HE staining showed that the hypothalamic neurons of WKY rats were arranged neatly and regularly, the nucleus was oval or round, there was deviation, and the nucleolus was clear. In the SHR hypothalamus, the eosinophilia of hypothalamic tissue was enhanced (staining was reddish), the number of cells decreased, the cell body and nucleus became larger, the number of cells edema increased, and the number of neurons decreased, which was accompanied by vacuolar degeneration. Compared with the SHR group, the cell morphology of the EU-TT group was improved to varying degrees after EU-TT administration (Figure 2A). This effect was also confirmed by electron microscopy (Figure 2B).

\section{Proteomics}

\section{Identification of differential proteins}

In order to study the differences in protein expression and to identify the drug targets in the hypothalami of SHRs after EU-TT administration, we conducted a label free high-throughput quantitative proteomics assay on hypothalamus tissue. A total of 2,796 differential protein points were detected in the hypothalami of SHRs after EU-TT treatment, among which 248 proteins were significantly different (157 proteins were increased and
91 proteins were decreased). The heatmap in Figure $3 \mathrm{~A}$ shows the differentially expressed (DE) proteins between the EU-TT and SHR group. The top 10 upregulated and downregulated proteins are summarized in Table 1.

\section{Bioinformatics analysis of $\mathrm{DE}$ proteins in the hypothalamus after EU-TT treatment}

Kyoto Encyclopedia of Genes and Genomes (KEGG) pathway analysis was performed to interpret the pharmacology of EU-TT in the central regulation of antihypertension from the perspective of DE proteins.

Compared with untreated SHRs, the MAPK signaling pathway (ID: map04010, $\mathrm{P}=0.0001$ ), the neurotrophin signaling pathway (ID: map04722, $\mathrm{P}=0.0019$ ), the VEGF signaling pathway (ID: map04370, $\mathrm{P}=0.0050$ ), the Ras signaling pathway (ID: map04014, $\mathrm{P}=0.0073$ ), and choline metabolism in cancer (ID: map05231, $\mathrm{P}=0.0134$ ) were the most significant pathways involved in the pharmacodynamic process of EU-TT treatment on the hypothalamus (Figure 3B). Among them, the MAPK signaling pathway was the most notable.

\section{$R T-q P C R$ results}

The results of RT-qPCR showed that the expression levels of $\mathrm{p} 38$, ERK, and JNK in the SHR group were significantly higher than those in the WKY group: p38 (19 times), ERK (17 times), and JNK (19 times). EU-TT treatment significantly reduced these indicators compared with the SHR group: p38 (1.5 times), ERK (1.8 times), and JNK (1.5 

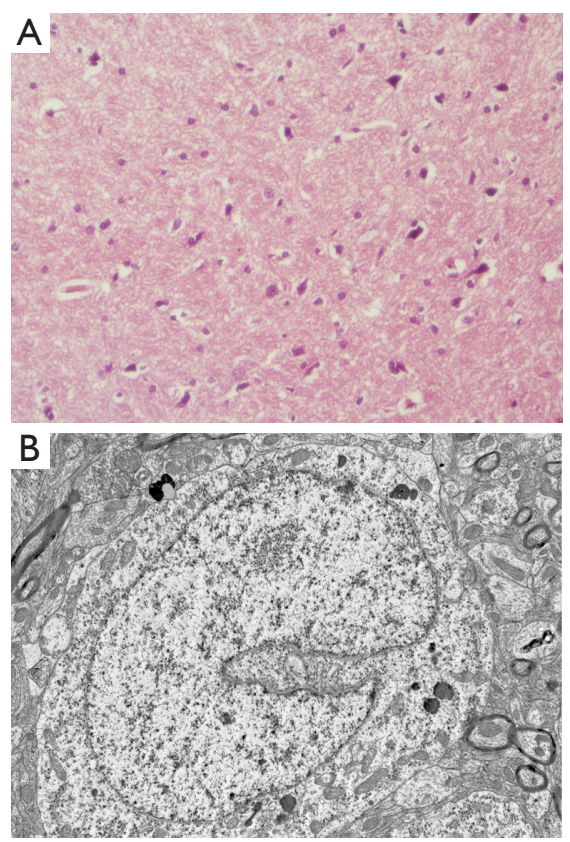

WKY
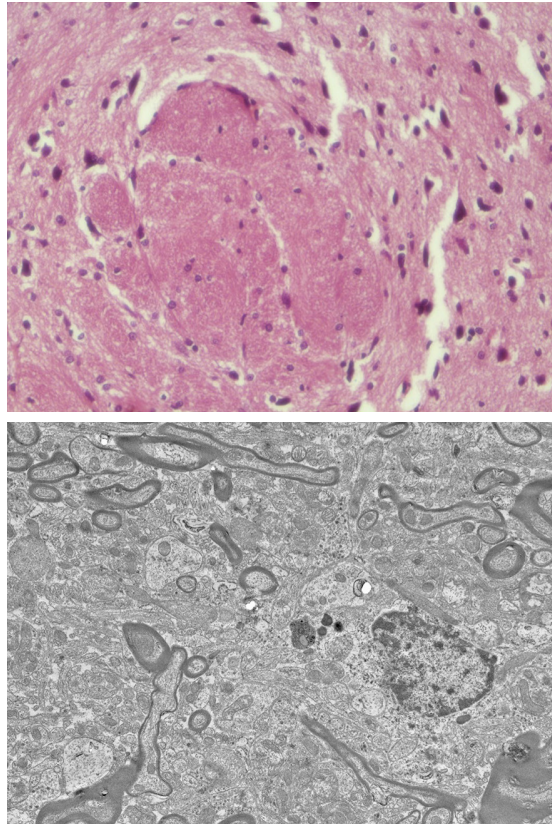

SHR
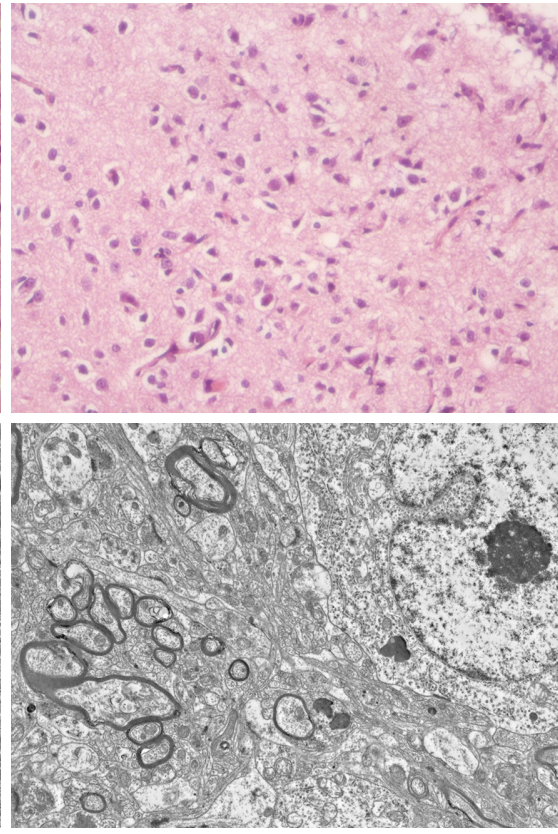

EU-TT

Figure 2 EU-TT improves the morphology of the hypothalamus in SHRs. (A) Representative images of HE staining ( $\times 200$ ); (B) representative images of TEM (×15,000). EU-TT, Eucommia ulmoides-Tribulus terrestris; SHRs, spontaneously hypertensive rats.

A

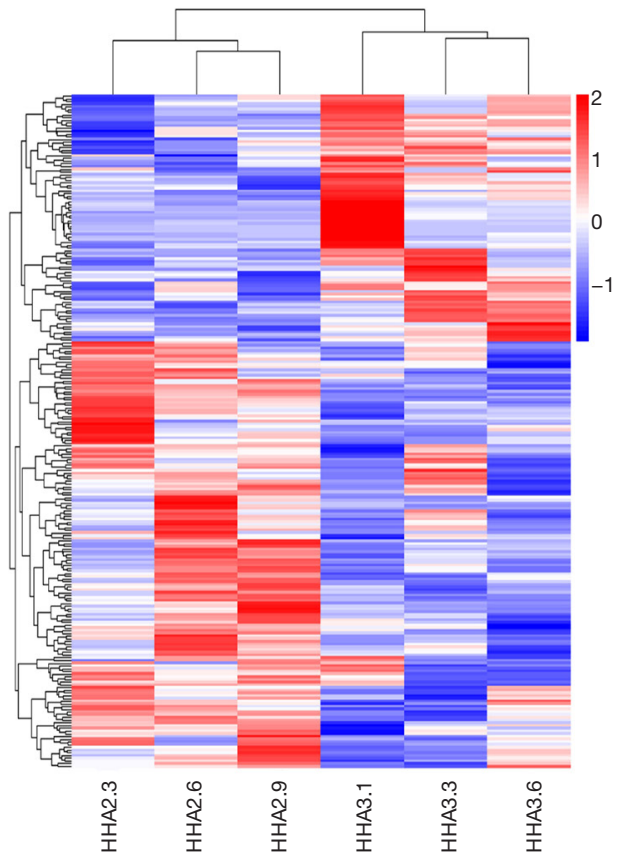

B

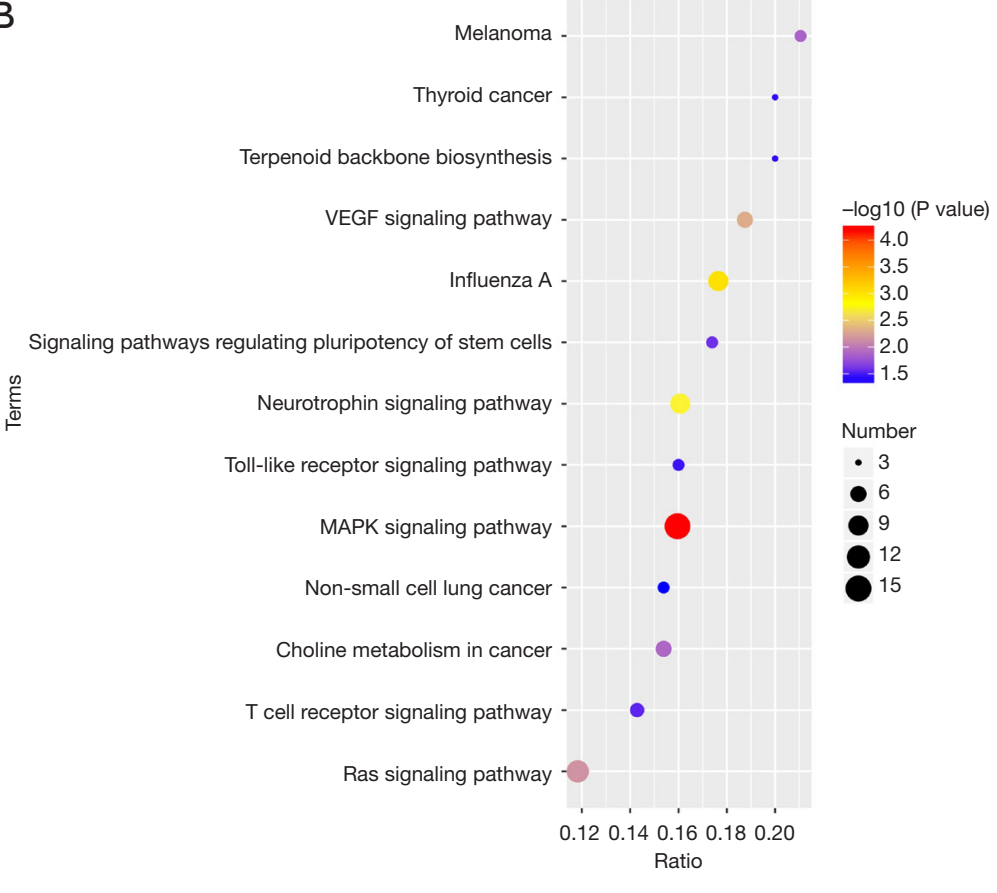

Figure 3 The target of EU-TT is the MAPK signaling pathway. (A) Heatmap shows the DE proteins between the EU-TT and SHR groups. HHA2: EU-TT group; HHA3: SHR group; (B) dot plot of KEGG enrichment for DE proteins (EU-TT vs. SHR). EU-TT, Eucommia ulmoides-Tribulus terrestris; SHR, spontaneously hypertensive rat; DE, differentially expressed; KEGG, Kyoto Encyclopedia of Genes and Genomes. 
Table 1 Top 10 upregulated and downregulated proteins determined by the label free proteomics assay (n=3, EU-TT vs. SHR)

\begin{tabular}{|c|c|c|c|c|}
\hline Gene name & Regulation & $P$ values & log2 fold change & Description \\
\hline Acan & Up & 0.0310 & 2.7164 & Aggrecan core protein \\
\hline Celsr1 & Up & 0.0068 & 2.5926 & Protein Celsr1 \\
\hline Rp/23 & Up & 0.0259 & 2.4145 & $60 S$ ribosomal protein $\mathrm{L} 23$ \\
\hline Magee1 & Up & 0.0036 & 2.2187 & Melanoma-associated antigen E1 \\
\hline Ahnak & Up & 0.0382 & 2.2115 & Protein Ahnak \\
\hline Coq9 & Up & 0.0030 & 2.1609 & Ubiquinone biosynthesis protein COQ9, mitochondrial \\
\hline Apool & Up & 0.0476 & 2.0474 & MIC \\
\hline $\operatorname{ltga} 6$ & Down & 0.0001 & -1.7714 & Integrin, alpha 6, isoform CRA_a \\
\hline Bcr & Down & 0.0000 & -1.7524 & Protein Bcr \\
\hline Eif3g & Down & 0.0000 & -1.5212 & Eukaryotic translation initiation factor 3 subunit $\mathrm{G}$ \\
\hline Cpeb2 & Down & 0.0001 & -1.5058 & Protein Cpeb2 \\
\hline Actn2 & Down & 0.0018 & -1.4874 & Protein Actn2 \\
\hline Vps51 & Down & 0.0000 & -1.4400 & Protein Vps51 \\
\hline Ptbp1 & Down & 0.0017 & -1.3508 & Polypyrimidine tract binding protein 1 , isoform CRA_C \\
\hline Sarm1 & Down & 0.0000 & -1.2936 & Protein Sarm1 \\
\hline
\end{tabular}

EU-TT, Eucommia ulmoides-Tribulus terrestris; SHR, spontaneously hypertensive rat.

times, Figure 4A).

\section{Western blotting analysis}

The Western blot assay confirmed that EU-TT treatment decreased the protein expression of p-p38 (0.4 times), p-ERK (0.6 times), and p-JNK (0.8 times) compared with the SHR group, which was consistent with the data of RT-qPCR and in line with the KEGG pathway analysis (Figure 4B,4C).

\section{Discussion}

The hypothalamus, which is located in the ventral forebrain, plays important roles in regulating energy homeostasis, fluid balance, stress, growth, reproductive behavior, emotion, and circadian rhythms (12). Abnormal development or function of the hypothalamus leads to many diseases in humans, such as growth defects, obesity, diabetes mellitus and insipidus, hypertension, and amenorrhea (13-18). Many animal studies have demonstrated that an abnormal paraventricular nucleus of the hypothalamus (PVH) is highly related to the pathogenesis of hypertension $(19,20)$.

Proteomics technology can intuitively show the regulation of Traditional Chinese Medicine at the protein level, and is an effective tool to find drug targets. We used proteomics to accurately and deeply study the action mechanism of EU-TT, which provided a favorable research strategy for clarifying the action mechanism of EU-TT. Both Eucommia ulmoides and Tribulus terrestris are commonly used in combination in the treatment of cardiovascular and cerebrovascular diseases. The effect of EU-TT in improving the hypothalamic function of aging SHRs was investigated in the present study. Eight-week administration 

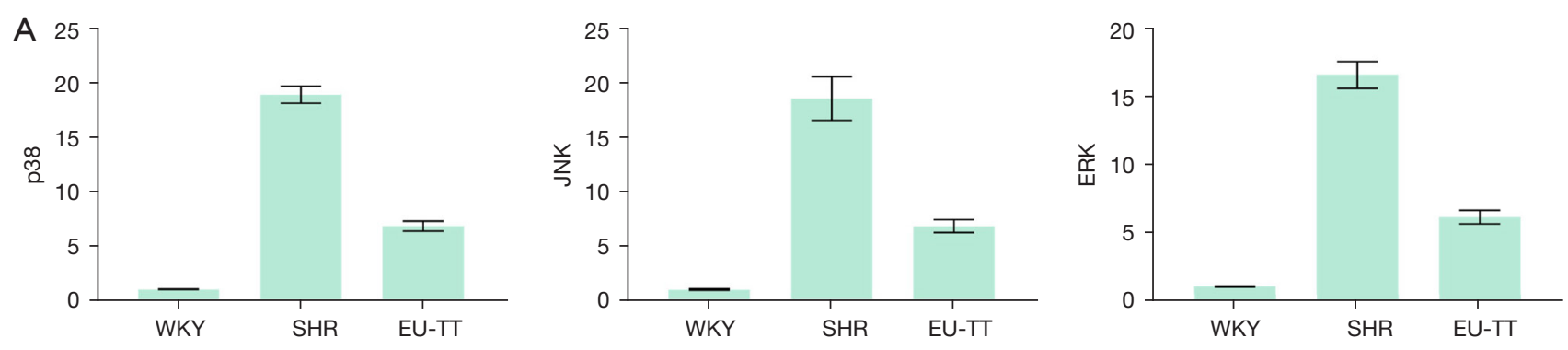

B
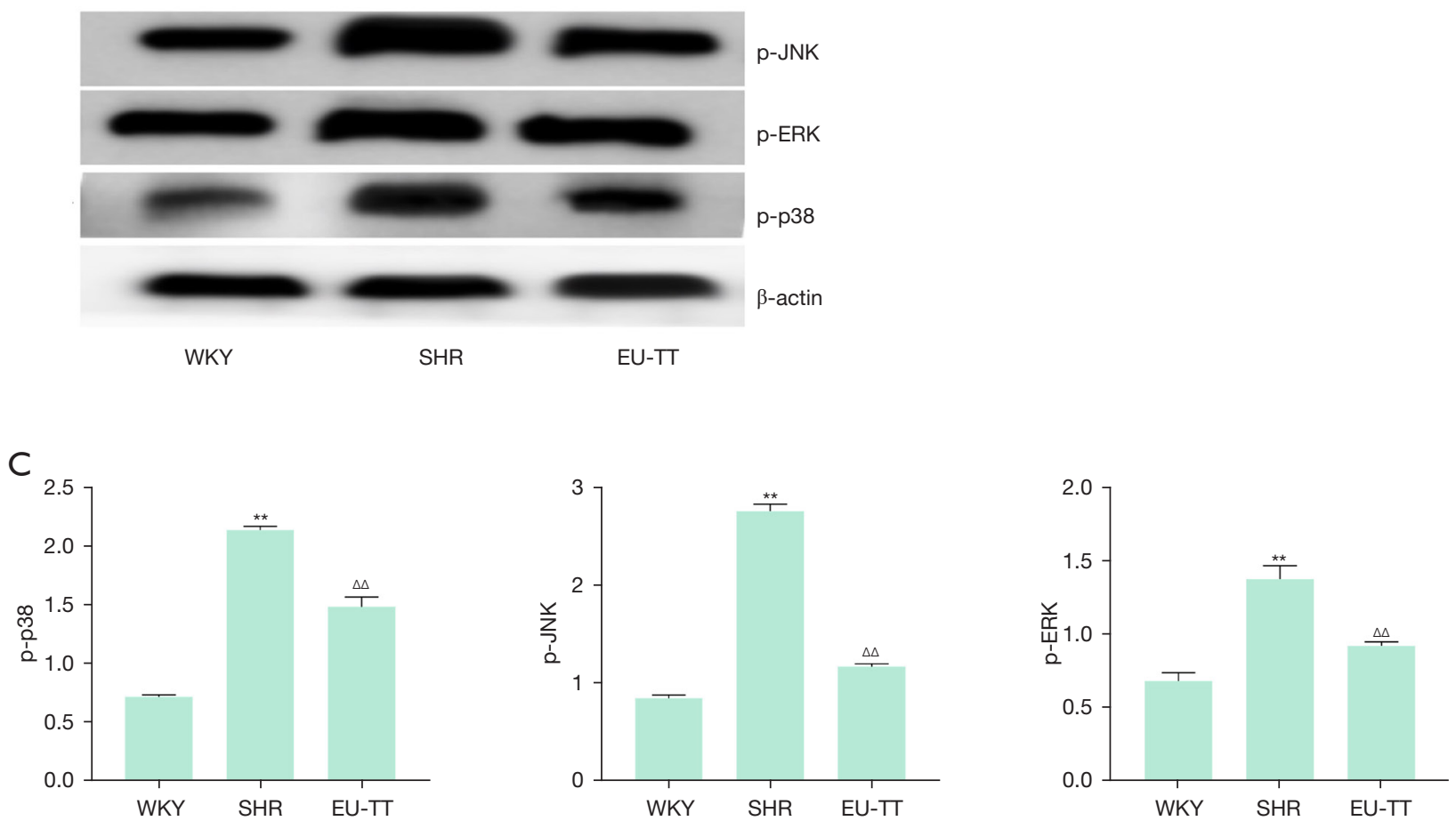

Figure 4 RT-qPCR and Western blotting analysis. (A) The mRNA expression levels determined by RT-qPCR. The value of the y-axis represents the multiple of each group and the WKY group. ERK (multiple), JNK (multiple), and p-38 (n=3). (B) Representative images: Western blot assay of p-ERK, p-JNK, and p-p38. (C) The analysis of WB light density was performed using Image J for p-ERK, p-JNK, and p-p38 (n=3). ${ }^{* *} \mathrm{P}<0.01$ vs. the WKY group; ${ }^{\Delta \Delta} \mathrm{P}<0.01$ vs. the SHR group. RT-qPCR, reverse transcription-quantitative polymerase chain reaction; WKY, Wistar-Kyoto rats; SHR, spontaneously hypertensive rat; EU-TT, Eucommia ulmoides-Tribulus terrestris.

of EU-TT led to a successful decrease of SBP and DBP in aging SHRs. EU-TT treatment also improved the morphology of the hypothalamus in SHRs, which was demonstrated by HE staining and electron microscopy.

We focused on hypothalamic composition and screened the targets of EU-TT in the hypothalamus by the proteomics assay. According to the $248 \mathrm{DE}$ proteins, the MAPK signaling pathway was the most significant pathway involved in the effect of EU-TT on the hypothalamus.

EU-TT significantly decreased the expression of p-p38, p-ERK, and p-JNK in the hypothalami of SHRs, which confirmed the results of the proteomics assay and suggested that EU-TT inhibited the activity of the MAPK signaling pathway. Three major MAPK families, including the extracellular response kinase (ERK), c-Jun N-terminal kinase (JNK), and p38, are widely involved in mammalian systems (21). JNK participates in signal transduction induced by various stressors, including autophagy and apoptosis (22). ERK is distributed in various tissues and is involved in the regulation of cell proliferation and differentiation (23), 
whereas p38 is considered to regulate inflammation and apoptosis (24), thus becoming a target for the development of anti-inflammatory drugs. It is known that the MAPK signaling pathway remains activated during the pathological process of hypertension. EU-TT significantly decreased the expression of p-p38, p-ERK, and p-JNK in the hypothalami of SHRs, which suggested that EU-TT exerts its role in the central regulation of antihypertension in the hypothalamus by targeting the MAPK signaling pathway. A major characteristic of Chinese medicine is its multitarget approach, often working through different pathways. In this study, we did not obtain the effective component combination of EU-TT, which provides some suggestions for future research.

In conclusion, the present investigation confirms the antihypertensive effect of EU-TT and suggests that the MAPK signaling pathway might be the target of EU-TT in the hypothalami of SHRs.

\section{Acknowledgments}

Funding: The study was supported by the National Natural Science Foundation of China (No. 82174177), and Shandong Province 'Taishan Scholar' Construction Project Funds (No. 2018-35).

\section{Footnote}

Reporting Checklist: The authors have completed the ARRIVE reporting checklist. Available at https://dx.doi. org/10.21037/atm-21-6390

Data Sharing Statement: Available at https://dx.doi. org/10.21037/atm-21-6390

Conflicts of Interest: All authors have completed the ICMJE uniform disclosure form (available at https://dx.doi. org/10.21037/atm-21-6390). The authors have no conflicts of interest to declare.

Ethical Statement: The authors are accountable for all aspects of the work in ensuring that questions related to the accuracy or integrity of any part of the work are appropriately investigated and resolved. Animal welfare and experimental steps were executed in accordance with the principles of "Laboratory animal-Guidelines for ethical review of animal welfare (GB/T 35892-2018)" (General Administration of Quality Supervision, Inspection and
Quarantine of the People's Republic of China, 2018), and approved by the Faculty of Animal Ethics Committee of Affiliated Hospital of Shandong University of Traditional Chinese Medicine (Jinan, China) (No. 2020-08).

Open Access Statement: This is an Open Access article distributed in accordance with the Creative Commons Attribution-NonCommercial-NoDerivs 4.0 International License (CC BY-NC-ND 4.0), which permits the noncommercial replication and distribution of the article with the strict proviso that no changes or edits are made and the original work is properly cited (including links to both the formal publication through the relevant DOI and the license). See: https://creativecommons.org/licenses/by-nc-nd/4.0/.

\section{References}

1. Li J, Fan L, Hua Q, et al. Chinese guidelines for the management of hypertension in the elderly 2019. Chinese Journal of Multiple Organ Diseases in the Elderly 201;18:81-106.

2. Liu GS. Chinese guidelines for the prevention and treatment of hypertension 2010. Chinese Journal of Medical Frontier (Electronic Edition) 2011;3:42-93.

3. Tarride JE, Lim M, DesMeules M, et al. A review of the cost of cardiovascular disease. Can J Cardiol 2009;25:e195-202.

4. Fadini GP, Ceolotto G, Pagnin E, et al. At the crossroads of longevity and metabolism: the metabolic syndrome and lifespan determinant pathways. Aging Cell 2011;10:10-7.

5. Safar ME. Arterial aging--hemodynamic changes and therapeutic options. Nat Rev Cardiol 2010;7:442-9.

6. Guo Y, Shi DZ, Yin HJ, et al. Effects of Tribuli saponins on ventricular remodeling after myocardial infarction in hyperlipidemic rats. Am J Chin Med 2007;35:309-16.

7. Jiang $\mathrm{YH}, \mathrm{Guo} \mathrm{JH}, \mathrm{Wu}$ S, et al. Vascular protective effects of aqueous extracts of Tribulus terrestris on hypertensive endothelial injury. Chin J Nat Med 2017;15:606-14.

8. Jiang $\mathrm{YH}$, Yang $\mathrm{CH}, \mathrm{Li} \mathrm{W}$, et al. Aqueous extracts of Tribulus terrestris protects against oxidized low-density lipoprotein-induced endothelial dysfunction. Chin J Integr Med 2016;22:193-200.

9. Qi YZ, Yang XS, Jiang YH, et al. Study of the Mechanism Underlying the Antihypertensive Effects of Eucommia ulmoides and Tribulus terrestris Based on an Analysis of the Intestinal Microbiota and Metabonomics. Biomed Res Int 2020;2020:4261485. 
10. Qi YZ, Jiang YH, Jiang LY, et al. Effect of Eucommia ulmoides - Tribulus terrestris on intestinal microbiome of aged spontaneously hypertensive rats. Chin J Hypertens 2019;27:454-62.

11. Qi YZ, Jiang YH, Yang CH. Study on fecal metabolomics of Eucommia ulmoides - Tribulus terrestris on reducing blood pressure in elderly spontaneously hypertensive rats based on non targeted gas chromatography-mass spectrometry. Chinese Journal of Traditional Chinese Medicine 2020;38:131-4, 288-9.

12. Saper CB, Lowell BB. The hypothalamus. Curr Biol 2014;24:R1111-6.

13. Morton GJ, Meek TH, Schwartz MW. Neurobiology of food intake in health and disease. Nat Rev Neurosci 2014;15:367-78.

14. Cryer PE. Mechanisms of hypoglycemia-associated autonomic failure and its component syndromes in diabetes. Diabetes 2005;54:3592-601.

15. de Wardener HE. The hypothalamus and hypertension. Physiol Rev 2001;81:1599-658.

16. Vescovi JD, Jamal SA, De Souza MJ. Strategies to reverse bone loss in women with functional hypothalamic amenorrhea: a systematic review of the literature. Osteoporos Int 2008;19:465-78.

17. Feng S, Xing C, Shen T, et al. Abnormal Paraventricular Nucleus of Hypothalamus and Growth Retardation Associated with Loss of Nuclear Receptor Gene COUPTFII. Sci Rep 2017;7:5282.

Cite this article as: Zhang $\mathrm{P}$, Zhang $\mathrm{J}$, Wei J, Feng S, Qi Y, Yang C, Jiang Y. Proteomics reveals the role of Eucommia ulmoides-Tribulus terrestris in the central regulation of antihypertension by improving the hypothalamus in spontaneously hypertensive rats. Ann Transl Med 2022;10(1):19. doi: 10.21037/atm-21-6390
18. Leroy C, Karrouz W, Douillard C, et al. Diabetes insipidus. Ann Endocrinol (Paris) 2013;74:496-507.

19. Sun HJ, Chen D, Han Y, et al. Relaxin in paraventricular nucleus contributes to sympathetic overdrive and hypertension via PI3K-Akt pathway. Neuropharmacology 2016;103:247-56.

20. Zhang F, Sun HJ, Xiong XQ, et al. Apelin-13 and APJ in paraventricular nucleus contribute to hypertension via sympathetic activation and vasopressin release in spontaneously hypertensive rats. Acta Physiol (Oxf) 2014;212:17-27.

21. Zhang G, Li J, Purkayastha S, et al. Hypothalamic programming of systemic ageing involving IKK- $\beta, \mathrm{NF}-\kappa \mathrm{B}$ and GnRH. Nature 2013;497:211-6.

22. Ding HS, Huang Y, Chen Z, et al. Regulator of G-protein signalling 5 deficiency impairs ventricular remodelling after myocardial infarction by promoting NF- $\kappa \mathrm{B}$ and MAPK signalling in mice. Biochem Biophys Res Commun 2018;499:143-9.

23. Sun Y, Liu WZ, Liu T, et al. Signaling pathway of MAPK/ ERK in cell proliferation, differentiation, migration, senescence and apoptosis. J Recept Signal Transduct Res 2015;35:600-4.

24. Yokota T, Wang Y. p38 MAP kinases in the heart. Gene 2016;575:369-76.

(English Language Editor: C. Betlzar) 